\title{
Educating military primary health-care providers in genomic medicine: lessons learned from the MilSeq Project
}

\author{
Megan D. Maxwell, MS, LCGC (10) ${ }^{1,2,3,4,5}$, Rebecca Hsu, BA ${ }^{6}$, Rubaiya Islam, $\mathrm{MS}^{6}$, Jill O. Robinson, $\mathrm{MA}^{6}$, \\ Stacey Pereira, PhD ${ }^{6}$, Cubby L. Gardner, PhD, FNP-C ${ }^{7}$, Robert C. Green, MD, MPH ${ }^{4,5,8}$, \\ MilSeq Project, and Mauricio De Castro, MD, AACMG $^{9}$
}

Purpose: With few trained genetics professionals, the Military Health System is ill-equipped to manage the rapid expansion of genomic medicine. The MilSeq Project introduces an alternative service delivery model (ASDM) in which primary health-care providers (HCPs) provide post-test counseling (PTC) to healthy Airmen who have undergone exome sequencing. We describe HCP performance after a prerequisite educational intervention (EI).

Methods: After a brief EI and pre-/posteducation surveys, HCPs were eligible to provide PTC with a genetic counselor available for consult. PTC was recorded, transcribed, and reviewed. Opportunities for improvement were organized into four error adjustment categories: (1) knowledge limitation, (2) minor, (3) moderate, and (4) critical. Thematic analysis was also performed.

Results: Pre-/posteducation survey responses revealed statistically significant improvements in all domains. Minor error adjustments were most represented $(n=93)$, followed by knowledge limitation $(n=39)$ and moderate $(n=19)$. No critical errors were identified, and 17 transcripts required no adjustment. Thematic analysis revealed four themes that would benefit from more focused education: (1) family-centered care, (2) conveying risk, (3) disease knowledge, and (4) assay knowledge.

Conclusion: HCPs demonstrated competence in basic PTC after a brief EI. This ASDM may be a viable interim response to the shortage of genetics professionals in some systems.

Genetics in Medicine (2020) 22:1710-1717; https://doi.org/10.1038/s41436020-0865-7

Keywords: genetic counseling; genetic services; education; intervention; exome sequencing

\section{INTRODUCTION}

As genomic sequencing (GS) has broadened the scope and relevance of genetic medicine, the demand for expert clinical interpretation exceeds the supply of trained genetic professionals. Prominent medical genetics organizations have recognized this growing shortage and have initiated nationwide workforce analyses to address the identified gap. ${ }^{1,2}$ Assuming approximately $70 \%$ projected career growth and a stable supply-to-demand ratio of 1 genetic counselor (GC) to 75,000 individuals, the Genetic Counselor Workforce Working Group estimates that the GC shortage in the United States may not equilibrate for another decade. ${ }^{2}$

The demand for genetic services in the US military has increased in tandem with the civilian sector. ${ }^{3}$ With few trained genetic professionals in its midst, the US military is currently ill-equipped to manage the expanding role of genetic medicine in routine health-care delivery. For this reason, military health-care providers (HCPs) with training in basic pre- and post-test genetic counseling are crucial, particularly with respect to appreciating the nuances and specific concerns of military service. ${ }^{4}$
The MilSeq Project (Enabling Personalized Medicine through Exome Sequencing in the US Air Force) was designed to study the benefits and risks of exome sequencing (ES) implementation into the Military Health System (MHS). A component of its development was intended to explore an alternative service delivery model as an interim response to the shortage of genetics professionals in the near term. Because the MHS is one of the largest and most complex health-care institutions, with millions of beneficiaries and hundreds of military treatment facilities (MTFs) worldwide, ${ }^{5}$ traditional and nontraditional (e.g., telegenetics, group counseling) models have not been sufficient to provide genetics services to the totality of its members.

The MilSeq Project introduces an iteration of the alternative service delivery model first piloted in the MedSeq Project, ${ }^{6-9}$ in which the primary care workforce provides basic post-test genetic counseling. To minimize a "substitution effect", the MilSeq model includes a prerequisite GC-instructed educational intervention, point-of-care support and ongoing supervision by trained genetics professionals, and postcounseling evaluation. Here we describe HCP performance in their

\footnotetext{
${ }^{1}$ University Health System, San Antonio, TX, USA; ${ }^{2}$ University of Texas Health Science Center, San Antonio, TX, USA; ${ }^{3}$ Lackland Air Force Base, San Antonio, TX, USA; ${ }^{4}$ Brigham and Women's Hospital, Boston, MA, USA; ${ }^{5}$ Harvard Medical School, Boston, MA, USA; ${ }^{6}$ Baylor College of Medicine, Houston, TX, USA; ${ }^{7}$ US Army Medical Research and Development Command, Fort Detrick, MD, USA; ${ }^{8}$ Broad Institute of MIT and Harvard, Cambridge, MA, USA; ${ }^{9}$ Air Force Medical Genetics Center, Keesler Air Force Base, Biloxi, MS, USA. Correspondence: Megan D. Maxwell (Megan.Maxwell@uhs-sa.com)
}

Submitted 14 January 2020; revised 31 May 2020; accepted: 2 June 2020

Published online: 10 July 2020 
provision of basic genetic counseling services to 75 ostensibly healthy active-duty Airmen who underwent clinical ES.

\section{MATERIALS AND METHODS}

\section{Ethics statement}

The MilSeq Project protocol, which details study rationale, objectives, design, methodology, anticipated analyses, and ethical considerations, was reviewed and approved by the US Air Force 59th Medical Wing Institutional Review Board (59 MDW IRB) in May 2017. The 59 MDW IRB is a fully accredited member of the Association for the Accreditation of Human Research Protection Programs. The voluntary, fully informed consent of the subjects used in this research was obtained as required by 32 CFR 219 and AFI 40-402, Protection of Human Subjects in Biomedical and Behavioral Research. Study details may be publicly accessed at ClinicalTrials.gov with MilSeq identifier: NCT03276637.

\section{Health-care provider recruitment}

A nonrandomized convenience sample of 3 staff internists, 5 resident internists, 1 staff preventive medicine physician, 1 staff aerospace medicine physician, and 2 trainee health nurse practitioners $(n=12)$ were recruited by group announcement and personal advertisement at a single US military installation. All HCP participants were active-duty Air Force. As a function of study design, the HCP cohort was small relative to the size of the patient cohort to maximize $\mathrm{HCP}$-patient interaction.

\section{Patient recruitment}

A nonrandomized convenience sample of active-duty Airmen $(n=93)$ were recruited by flyer, newsletter, social media posting, and personal advertisement in proximity to primary care clinics at a single US military installation. In phase I of recruitment, Airmen were asked to complete a baseline survey in electronic format, designed to assess knowledge, attitudes, and perceptions of GS. The Preferences Instrument for Genomic Secondary Results (PIGSR) tool was also incorporated to examine preference patterns for GS disclosure. ${ }^{10}$ Preference patterns examined were (1) disclosure of all findings from childhood-onset to adult-onset conditions, (2) all findings excluding one condition of heightened concern, (3) conditions relevant only to oneself, (4) conditions relevant only to offspring, and (5) more complex disclosure preferences. The baseline survey concluded with an invitation to participate in a second phase that involved clinical ES. Airmen electing to advance to phase II $(n=75)$ were scheduled for an informed consent session with the licensed and certified GC project manager, during which they were informed of the potential risks, benefits, and outcomes of participation. Prospective participants were advised that all identified pathogenic and likely pathogenic variants would be disclosed irrespective of indicated PIGSR preference, including findings for which there are currently no preventive measures, risk reduction options, treatments, or cures available, such as the APOE risk allele associated with
Alzheimer disease (which was specifically cited as an example). The main categories of findings to be returned were outlined: (1) monogenic disease and autosomal recessive carrier status for more than 5000 genes, (2) established risk alleles with odds ratios $>3$ for 8 common conditions (e.g., CHEK2-associated breast cancer risk, pulmonary fibrosis, Alzheimer disease), and (3) 230 pharmacogenomic (PGx) variants with $\mathrm{CPIC}^{\circledast}$ and PharmGKB Clinical Annotation levels $\mathrm{A}$ and $1 \mathrm{~A} / 1 \mathrm{~B}$, respectively. They were further advised that variants of uncertain significance would not be reported unless later reclassified as likely pathogenic or pathogenic, at which time a revised report would be issued as appropriate. Following voluntary informed consent, patient participants provided blood samples for ES and later received customized exome reports with interpretation and result disclosures performed by HCP participants. The patient cohort was intentionally unselected for Air Force occupation.

\section{Educational intervention development and instruction}

An educational intervention tailored to the needs of the US military was developed by an MHS-experienced licensed and certified GC and active-duty clinical geneticist. The educational module was further reviewed and curated by a larger, multidisciplinary team of research GCs, clinical geneticists, molecular geneticists, primary HCPs (civilian and military), bioinformaticians, bioethicists, and legal experts.

For consistency with standard medical genetics curricula, educational content was principally derived from Medical Genetics. ${ }^{11}$ Content was balanced between fundamental genetics topics (e.g., Mendelian inheritance, Punnett square crosses, pedigree nomenclature) and nuanced, anticipatory guidance for study-related post-test counseling (e.g., expressivity/penetrance, compound heterozygosity, PGx). Structured common and complex clinical vignettes representative of each inheritance pattern were presented to exercise clinical reasoning. Characteristics unique to genetic test utilization within MTFs were also presented, including but not limited to (1) electronic health record (EHR) system navigation, (2) laboratory capitation, and (3) internal versus external (i.e., MTF/telegenetics versus civilian) genetic referral logistics. Basic genetic principles were minimally modified from the source material ${ }^{11}$ while more conceptual learning objectives such as clinical perception, interpretation, and gestalt required considerable empirical supplementation. As primary HCPs' availability for continuing education can be limited by administrative and clinical obligations, ${ }^{12}$ the module was developed to be instructed in a 3-hour period and approved for three American Medical Association Physician Recognition Award Category 1 Credits $^{\mathrm{m}}$. HCP participants also received clinic aids, which included counseling prompts and common motifs associated with autosomal dominant, autosomal recessive, and X-linked inheritance.

\section{Survey assessment}

After obtaining informed consent, HCP participants were asked to complete pre-education surveys designed to assess their 
perceived self-confidence, preparedness, self-efficacy, and knowledge with regard to the provision of genetic services. Immediately following instruction, posteducation surveys were administered to assess any change in these metrics.

Five items measured HCP participants' confidence, with response options on a 4-point Likert-type scale anchored on one end with $1=$ "not at all confident" and the other end with $4=$ "very confident". ${ }^{13}$ Preparedness was assessed by asking, "How prepared do you feel to disclose results directly to patients?" using a 4-point Likert-type scale with response options from $1=$ "very prepared" to $4=$ "not at all prepared." ${ }^{\prime 9}$ HCPs' self-efficacy was measured by seven items using a 5-point Likert scale with response options anchored on one end by $1=$ "strongly disagree" and the other end with $5=$ "strongly agree" (Table 1). ${ }^{14,15}$ Genetic knowledge was assessed using 14 multiple-choice items adapted from the Health Literacy INDEX ${ }^{14}$ and the Genetics Literacy Assessment Instrument. ${ }^{16}$ Select questions from these validated tools were presented with a mock exome report for reference. ${ }^{15-17}$ Descriptive statistics, including means, standard deviations, medians, and interquartile ranges, were calculated to characterize HCP participant data. Average scores were

Table 1 MilSeq health-care provider self-confidence, preparedness, and self-efficacy.

\begin{tabular}{|c|c|c|c|c|}
\hline Item(s) & Self-confi & responder & & \\
\hline Genomic sequencing knowledge & Not at all & Not very & Somewhat & Very \\
\hline Pre-education & 5 & 5 & 2 & 0 \\
\hline Posteducation & 0 & 0 & 10 & 2 \\
\hline Explain a pathogenic result & Not at all & Not very & Somewhat & Very \\
\hline Pre-education & 2 & 7 & 3 & 0 \\
\hline Posteducation & 0 & 1 & 8 & 3 \\
\hline Recommendations for a pathogenic result & Not at all & Not very & Somewhat & Very \\
\hline Pre-education & 4 & 6 & 2 & 0 \\
\hline Posteducation & 0 & 0 & 9 & 3 \\
\hline Understand and disclose an exome report & Not at all & Not very & Somewhat & Very \\
\hline Pre-education & 5 & 7 & 0 & 0 \\
\hline Posteducation & 0 & 0 & 7 & 5 \\
\hline
\end{tabular}

Item(s)

Preparedness: \# of respondents by Likert option $(n=12)$

\begin{tabular}{lllll}
\hline How prepared do you feel to disclose results? & Very prepared & Prepared & Slightly prepared & Not at all prepared \\
Pre-education & 1 & 0 & 6 & 5 \\
Posteducation & 2 & 10 & 0 & 0 \\
\hline
\end{tabular}

$\operatorname{Item}(\mathrm{s})$

Self-efficacy: \# of respondents by Likert option $(n=12)$

\begin{tabular}{|c|c|c|c|c|c|}
\hline Understand a genomic sequencing result & Strongly disagree & Disagree & Neither agree nor disagree & Agree & Strongly agree \\
\hline Pre-education & 2 & 7 & 2 & 1 & 0 \\
\hline Posteducation & 0 & 0 & 1 & 10 & 1 \\
\hline Obtain information to understand a result's significance & Strongly disagree & Disagree & Neither agree nor disagree & Agree & Strongly agree \\
\hline Pre-education & 2 & 4 & 5 & 1 & 0 \\
\hline Posteducation & 0 & 0 & 1 & 9 & 2 \\
\hline Understand a result's effect on patient health & Strongly disagree & Disagree & Neither agree nor disagree & Agree & Strongly agree \\
\hline Pre-education & 1 & 4 & 3 & 4 & 0 \\
\hline Posteducation & 0 & 0 & 1 & 11 & 0 \\
\hline Understand a result's influence on disease risk & Strongly disagree & Disagree & Neither agree nor disagree & Agree & Strongly agree \\
\hline Pre-education & 0 & 3 & 3 & 6 & 0 \\
\hline Posteducation & 0 & 0 & 1 & 9 & 2 \\
\hline Understand how genetic constitution may affect disease risk & Strongly disagree & Disagree & Neither agree nor disagree & Agree & Strongly agree \\
\hline Pre-education & 1 & 2 & 2 & 7 & 0 \\
\hline Posteducation & 0 & 0 & 0 & 11 & 1 \\
\hline Explain how genetic constitution may affect disease risk & Strongly disagree & Disagree & Neither agree nor disagree & Agree & Strongly agree \\
\hline Pre-education & 0 & 5 & 3 & 4 & 0 \\
\hline Posteducation & 0 & 0 & 0 & 11 & 1 \\
\hline Identify resources to obtain additional information & Strongly disagree & Disagree & Neither agree nor disagree & Agree & Strongly agree \\
\hline Pre-education & 2 & 5 & 1 & 4 & 0 \\
\hline Posteducation & 0 & 0 & 0 & 9 & 3 \\
\hline
\end{tabular}


computed for confidence and self-efficacy from each HCP's responses to all four confidence questions and all seven selfefficacy questions, respectively. The total number of correct responses to genetic knowledge questions were summed for each HCP to calculate an individual genetic knowledge score. Since the confidence, preparedness, and genetic knowledge scores were not normally distributed nor symmetric about the median, nonparametric sign tests were used to compare preand posteducation differences in these measures. Because the self-efficacy score was normally distributed, a paired $t$-test was used to compare pre- and posteducation differences in this measure. All statistical calculations were performed using $\mathrm{R}$ software. $^{18}$

\section{Performance analysis}

Upon completion of the prerequisite educational intervention, HCP participants were eligible to perform post-test counseling for patient participants with ongoing support and supervision from a Genome Resource Center (GRC), locally and remotely staffed by GCs, clinical geneticists, and molecular geneticists. Post-test counseling sessions were scheduled between HCPs and patients based on mutual availability. Scheduling was influenced by military-neutral factors, such as appointment accessibility and parental leave, but was also influenced by military-specific factors, such as training, deployment, and permanent change of station orders, resulting in varied experience (Table 2). Exome reports modeled after those developed for the MedSeq Project $^{7,19}$ were provided to HCP participants at least 1 week in advance of their scheduled appointments to allow sufficient time for review and GRC engagement as needed.

Table 2 MilSeq health-care provider result disclosures.

\begin{tabular}{|c|c|c|}
\hline $\begin{array}{l}\text { Health-care provider } \\
\text { identifier }\end{array}$ & $\begin{array}{l}\text { Health-care provider } \\
\text { credentials }\end{array}$ & $\begin{array}{l}\text { \# of } \\
\text { disclosures }\end{array}$ \\
\hline $\mathrm{HCP} 1^{\mathrm{a}}$ & Resident internist & 3 \\
\hline HCP 2 & $\begin{array}{l}\text { Preventive medicine } \\
\text { physician }\end{array}$ & 15 \\
\hline $\mathrm{HCP} 3^{\mathrm{b}}$ & Resident internist & 0 \\
\hline $\mathrm{HCP} 4^{\mathrm{a}}$ & Resident internist & 2 \\
\hline HCP 5 & Resident internist & 10 \\
\hline HCP 6 & Staff internist & 16 \\
\hline $\mathrm{HCP} 7^{\mathrm{C}}$ & $\begin{array}{l}\text { Aerospace medicine } \\
\text { physician }\end{array}$ & 5 \\
\hline $\mathrm{HCP} 8^{\mathrm{a}}$ & $\begin{array}{l}\text { Trainee health nurse } \\
\text { practitioner }\end{array}$ & 1 \\
\hline HCP $9^{d}$ & $\begin{array}{l}\text { Trainee health nurse } \\
\text { practitioner }\end{array}$ & 3 \\
\hline HCP 10 & Resident internist & 5 \\
\hline HCP 11 & Staff internist & 6 \\
\hline HCP 12 & Staff internist & 9 \\
\hline
\end{tabular}

At the time of post-test counseling, the GC project manager was embedded in clinic for ad hoc point-of-care consultation, and the 75 disclosures were audio recorded, transcribed, and reviewed by the GRC for accuracy and appropriateness. Transcript reviews were conducted by teleconference to facilitate inclusion of local and remote GRC members.

To standardize evaluation and minimize intrareviewer variability, a novel, adapted rubric was developed by modifying IRB regulations and guidelines pertaining to protocol deviation, violation, and adverse outcome reporting. ${ }^{20}$ Opportunities for improvement identified during posttest counseling sessions were organized into 4 categories: (1) knowledge limitation, lacking sufficient baseline knowledge and/or experience to comprehensively counsel the patient beyond the information provided in the report; (2) minor adjustments for errors posing minimal risk to optimal care; (3) moderate adjustments for errors posing some degree of suboptimal care; and (4) critical adjustments for errors endangering patient safety.

Adjustments were addressed as appropriate with individual HCP participants at the GRC's discretion and optional feedback was provided at the HCP participants' request.

\section{Thematic analysis}

Inductive thematic analysis was performed to identify patterns within the qualitative data. Transcribed disclosures were reviewed in aggregate and HCPs' post-test counseling was manually coded to capture gaps in functional knowledge and practice. Related codes were then collated for a general overview of recurrence, and the resulting framework was used to define common themes.

\section{RESULTS}

\section{Survey assessment}

Analysis of the HCP cohort's pre- and posteducation survey responses revealed statistically significant improvements from baseline $(P<0.05)$ in all domains assessed (Table 3$)$.

Table 3 MilSeq health-care provider domain assessment.

\begin{tabular}{lcl} 
Domains $(n=$ \# of items averaged) & \multicolumn{1}{l}{ Statistics } & \\
\hline Self-confidence $(n=4)$ & Mean (SD) & Paired sign test \\
\hline Pre-education & $1.8(0.5)$ & $P<0.001$ \\
\hline Posteducation & $3.3(0.4)$ & \\
Self-efficacy $(n=7)$ & Mean (SD) & Paired $t$-test \\
\hline Pre-education & $2.8(0.7)$ & $P<0.001$ \\
\hline Posteducation & $4.1(0.2)$ & \\
\hline Preparedness $(n=1)$ & Mean (SD) & Paired sign test \\
\hline Pre-education & $3.3(0.9)$ & $P=0.006$ \\
\hline Posteducation & $1.8(0.4)$ & \\
\hline Genetic knowledge $(n=14)$ & Mean (SD) & Paired sign test \\
Pre-education & $9.9(1.4)$ & $P=0.021$ \\
\hline Posteducation & $11.5(1.4)$ & \\
\hline
\end{tabular}




\section{Performance analysis}

Monogenic disease and carrier status variants were identified in $15 \%(n=11)$ and $87 \%(n=65)$ of patient participants, respectively, with an average of 2 and range of 0 to 6 carrier status variants per report. In $12 \% \quad(n=9)$ of patient participants, no variants associated with Mendelian conditions were identified. Risk alleles were returned in almost $50 \%$ $(n=37)$ of all reports and PGx variants were detected in $92 \%$ $(n=69)$ of patient participants, with an average of 4 and range of 0 to 8 medications per report.

GRC transcript review data demonstrate that the minor adjustment category, posing minimal risk to optimal patient care, was by far the most represented $(n=93)$, followed by knowledge limitation $(n=39)$ and moderate $(n=19)$ error adjustments, respectively (Table 4). These data yield an average rate of 1 to 2 minor error adjustments per transcript, and sporadic instance $(<1$ per transcript) of other adjustment types. However, increased $\mathrm{HCP}$-patient interaction was not correlated with reduced error rates. Of note is that no critical errors were identified, and that nearly one quarter (17/75; $23 \%$ ) of all transcripts required no GRC adjustment (Fig. 1).

\section{Thematic analysis}

Thematic analysis of systematic errors derived from transcript review revealed four major themes that would benefit from more focused education: (1) family-centered care, (2) conveying risk, (3) disease knowledge, and (4) assay knowledge.

\section{Family-centered care}

HCPs are traditionally trained in patient-centered care, in which the individual's needs and improved outcome drive health-care decisions. ${ }^{21}$ Therefore, they may overlook important counseling recommendations informed by family history. This was suggested by some providers' performance in contextualizing results within the pedigree, and in discussing cascade testing and implications for family members.

For example, a patient participant was identified to be heterozygous for a likely pathogenic variant in $L D L R$ (c.862G $>\mathrm{A}$ ), which is associated with autosomal dominant familial hypercholesterolemia $(\mathrm{FH})$. The HCP appropriately counseled the patient that he is affected with this highly penetrant condition, citing his most recent total cholesterol level of $300 \mathrm{mg} / \mathrm{dL}$ and prescribing a statin for medical

Table 4 MilSeq adjustment category organization: select transcript excerpts.

Adjustment category

Knowledge limitation

- Lacking sufficient baseline knowledge and/or experience to comprehensively counsel the patient beyond the information provided in the report

\section{Minor}

- Errors posing minimal risk to optimal care

\section{Moderate}

- Errors posing some degree of suboptimal care
Transcript excerpt

"What this would be testing for specifically is, [...] we know you have this gene: GJB2. [...] This is your mutation, right? So, we need to know, is her GJB2 gene affected?"

\section{"Yes, you have this one variant, but your}

husband, partner, would have to have the same

variant. [...] Let's say your husband just so

happened to also have the same mutation. [...]

There's a $25 \%$ chance that your child will not be affected, will get two good genes. There is a $50 \%$ chance that your child will be a carrier, just like you are. [...] Then there's a 25\% chance that your child would have the disease and would have two affected alleles on that gene."

"I'd seen that you were going for fertility treatments, is that correct? [...] And I noticed you were shorter in stature. So, Turner syndrome is a genetic condition in which one of your $X$ chromosomes is not there. Typically, these patients can't get pregnant at all, though. They have very kind of small ovaries that don't really release eggs. [...] Some women can have a partial Turner syndrome so to speak, but since you're pregnant probably unlikely."
Genome Resource Center rationale

The health-care provider understands autosomal recessive inheritance, counseling reproductive risk and offering carrier testing for the patient's spouse appropriately. However, the health-care provider offers carrier testing only for GJB2 and not GJB6, because he is unaware of the digenic relationship between the genes and it is omitted from the report.

The health-care provider understands autosomal recessive inheritance, counseling transmission appropriately. However, the health-care provider implies that the couple must have identical variants for reproductive risk, rather than any pathogenic variant in the same gene.

The health-care provider excludes a mosaic Turner syndrome differential diagnosis based on the patient's self-reported pregnancy, despite provocative clinical features such as significant short stature compared with first-degree relatives and documented fertility concerns. It was explained to the health-care provider that many women with mosaic Turner syndrome can achieve pregnancy but are still at risk for other syndromic health complications (e.g., cardiac) and may have some reproductive risk.

Not applicable. 


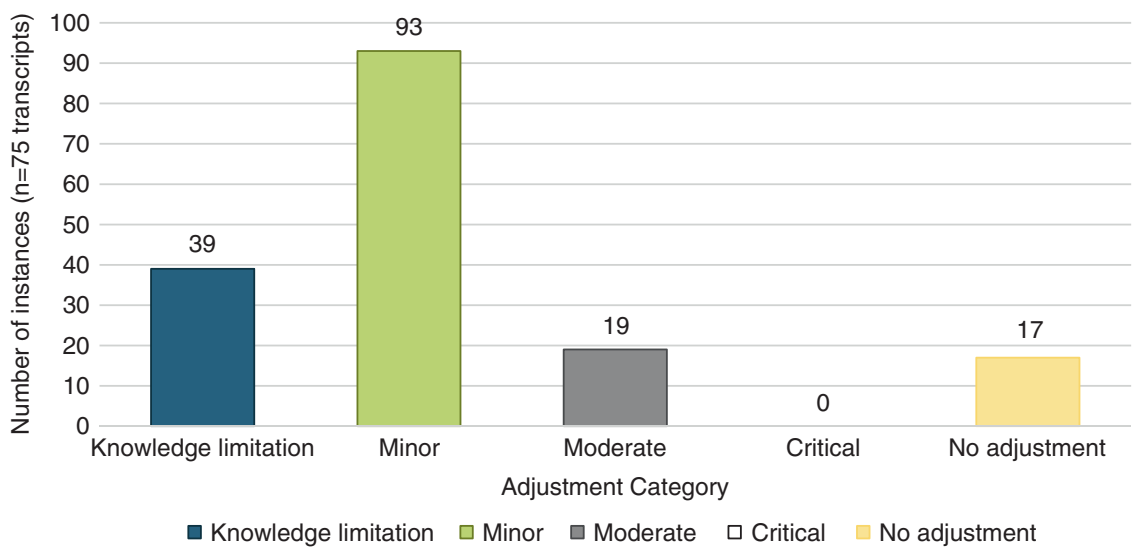

Fig. 1 MilSeq health-care provider performance.

management. However, the HCP did not address that the same health risks apply to at least one of the patient's biological parents and may apply to siblings due to the condition's autosomal dominant segregation and penetrance. The HCP also did not address the expert consensus for pediatric FH screening, which is relevant because the patient has two young sons for whom early pharmacologic therapy can be considered. ${ }^{22}$

\section{Conveying risk}

HCPs in routine medical practice treat common conditions and manage overall health. Therefore, they may experience challenges in distinguishing the rarity of monogenic conditions, and in conveying relative risk and multifactorial risk.

For example, a proportion of patient participants were identified to be heterozygous $(n=22)$ or homozygous $(n=3)$ for the established Alzheimer disease risk variant in $A P O E$ (c.388T $>$ C), also known as the $A P O E^{*} E 4$ allele. HCP participants who were assigned these exome reports for disclosure often discussed the associated odds ratios only superficially with their patients. While they appropriately counseled that the finding was not predictive of disease, but rather imparted increased risk, they often presented patients' relative risk arbitrarily rather than as a defined value calculated from the information provided in the report.

\section{Disease knowledge}

HCPs are generally less accustomed to the nuances of genetic conditions and genetic testing, and may be disinclined to use unfamiliar, genetic-specific resources such as GeneReviews ${ }^{\circledR}$ or Online Mendelian Inheritance in $\mathrm{Man}^{\circledast}$. This was evidenced by some providers' performance in describing disease phenotype and natural history.

For example, a patient participant was identified to be heterozygous for the reduced penetrance allele in HFE (c.187C $>\mathrm{G})$, which is associated with hereditary hemochromatosis $(\mathrm{HH})$. While the HCP explained the patient's carrier status, autosomal recessive inheritance, and reproductive risk correctly, he confused the pathophysiology of $\mathrm{HH}$ with polycythemia, counseling that the condition is characterized by increased erythropoiesis, elevated hematocrit, and thrombotic risk rather than iron overload, organ deposition, and iron-induced tissue damage. However, because therapeutic phlebotomy is common to both conditions, the patient was properly informed of primary treatment.

\section{Assay knowledge}

Most laboratory tests ordered in routine medical practice are chemical assays for which there are standard ranges and results are clearly defined as normal or abnormal. Therefore, the complexity of advanced, high-throughput next-generation sequencing assays with bioinformatic analysis may require more dedicated orientation.

For example, a patient participant was identified to be heterozygous for a pathogenic variant in FANCC (c.1642C >T), which is associated with autosomal recessive Fanconi anemia (FA). The patient reported his 24-month-old son to have multiple congenital café-au-lait spots and cystic hygroma, both of which have been linked to $\mathrm{FA},{ }^{23}$ as well as a negative neurofibromatosis (NF1) evaluation. Given this history, the HCP appropriately discussed carrier testing for the patient's spouse. However, this discussion was abandoned when the patient reported that his spouse had undergone direct-toconsumer (DTC) genetic testing through a company that advertises targeted FANCC analysis. The primary HCP was unaware of the distinction between DTC genotyping and clinical-grade GS, and was therefore unprepared to discuss the caveats of DTC genetic testing, such as the high false-positive rate requiring clinical confirmation, ${ }^{24}$ or false reassurance from a negative screen that does not exclude residual carrier risk. ${ }^{15}$

\section{DISCUSSION}

These preliminary data suggest that the investigated alternative service delivery model, in which genetics expertise is extended through GC- and geneticist-mediated primary care support, performed well in the MHS microcosm. As determined by a team of trained genetics professionals, HCP participants demonstrated competence in basic post-test genetic counseling after a brief, highly specific educational intervention and highly accessible onsite GC support. The majority of errors scrutinized 
were negligible to patients' general understanding of their result and overall health, requiring minimal adjustments. It is also noteworthy that no critical errors endangering patient safety, such as irreversible treatment decisions, were observed. In fact, most patient participants received accurate and appropriate medical management information.

When surveyed, all HCP participants reported statistically significant increases in self-confidence, preparedness, selfefficacy, and knowledge after the GC-instructed educational module-findings that were reinforced by their satisfactory counseling performance. While the HCP cohort was intentionally small, compensatory nonparametric analyses and lack of outliers indicate that the data set's statistical significance is valid despite small sample size.

That being said, the generalizability of these results should be interpreted judiciously, as the study was conducted in a closed health-care system with universal EHR integration and broad access to health-care resources, including a full-time licensed and certified GC dedicated exclusively to the project. Study materials were also developed to fulfill specific Air Force requirements and were almost exclusively dedicated to ES.

As it was not our objective to train primary HCPs to be as knowledgeable or effective as genetics professionals, gaps in functional knowledge and practice were expected and are regarded as opportunities for improvement. The educational intervention for this population was developed without precedent and further refinement was anticipated based on study outcomes.

Our real-world data demonstrate that HCP performance was largely predicted by educational content, which presented genomic concepts, approaches, and techniques much like they would be presented to a genetics student. This investigation suggests that learning experiences might be enhanced by prioritzing the HCPs' perspective when developing educational materials. Though opportunities for improvement are the focus here, it was also observed that HCP participants excelled in PGx post-test counseling; we hypothesize this is because drug metabolism is familiar, relatable, and intersects with their everyday practice. Building on this observation, it stands to reason that future versions of the educational module can be adapted to better leverage HCPs' existing expertise. Academic literature indicates that prior knowledge base and transfer from previous experiences is essential for long-term comprehension. ${ }^{25}$

Therefore, addressing considerations unique to the provision of genetics services in the primary care workforce would be expected to have greater success than continued emphasis on exact emulation. For example, rather than compound the complexity of relative risk with the incidence, prevalence, and odds ratios of rare genetic conditions, it may be more appropriate to first relate these topics to common conditions such as diabetes or heart disease before advancing. Understanding and interpreting these relationships will become increasingly important as medicine evolves toward personal risk scores, variable expressivity, and omics interactions, as all HCPs will be challenged to stratify multifactorial risk in the future. Also, because Mendelian conditions are infrequently encountered in primary care, it may be advantageous to combine didactics with additional exercises in clinical reasoning. Medical geneticists and genetic counselors in training refine their diagnostic process primarily via clinical practica, and primary HCPs do not have the benefit of such repeated clinical interaction. Therefore, beyond the clinical vignettes presented in our study, simulated patients can be introduced, permitting providers to familiarize themselves with online resources such as diagnostic checklists, toolkits, and/or clinic aids in a protected training environment. The opportunity to apply these tools in a mock clinical setting may encourage more consistent use in actual appointments, mitigating the adjustments required for cascade testing, disease, and assay knowledge.

It has been established that the shortage of trained genetics professionals has restricted access to genetic services due to either proximity or availability constraints. Consequently, there is a heightened demand for genetic services in routine medical practice and HCPs trained in other fields play an increasingly more active role in its provision. ${ }^{26,27}$ The alternative service delivery model introduced by the MilSeq Project may be a viable stopgap measure for the provision of genetics services within the context of this shortage, as the educational module piloted acknowledges HCPs' emerging role in genomic medicine with training that can facilitate partnership in practice. Such collaboration between primary HCPs and the few trained genetics professionals in the US military would triage genetic referrals, alleviating the demand and strain on these limited human resources. Based on our experience crafting and instructing a short educational intervention; providing real-time, point-of-care HCP consult; and retrospectively evaluating HCP performance, it is feasible to have nongenetics providers perform genetic counseling for some routine clinical indications with appropriate support.

\section{SUPPLEMENTARY INFORMATION}

The online version of this article (https://doi.org/10.1038/s41436020-0865-7) contains supplementary material, which is available to authorized users.

\section{ACKNOWLEDGEMENTS}

This material is based on research sponsored by Air Force Medical Support Agency, Research and Acquisition Directorate AFMSA SG5 under cooperative agreement number FA8650-17-2-6704. The US Government is authorized to reproduce and distribute reprints for Governmental purposes notwithstanding any copyright notation thereon. The views and conclusions contained herein are those of the authors and should not be interpreted as necessarily representing the official policies or endorsements, either expressed or implied, of Air Force Medical Support Agency, Research and Acquisition Directorate AFMSA/SG5 or the US Government. 


\section{DISCLOSURE}

R.C.G. receives advisor compensation from Alzheimer's Institute of America, Inc., Applied Therapeutics, Inc., Humanity, and Verily Life Sciences, LLC, and is cofounder of Genome Medical, Inc. The other authors declare no conflicts of interest.

Publisher's note Springer Nature remains neutral with regard to jurisdictional claims in published maps and institutional affiliations.

\section{REFERENCES}

1. American College of Medical Genetics and Genomics. ACMG applauds increase in funding for newborn screening and medical genetics workforce study in FY 2019 LHHS appropriations. https://www.acmg. net/PDFLibrary/Statement_\%20FY2019\%20LHHS\%20Appropriations_

10.10_Final.pdf. Accessed 6 July 2019.

2. Hoskovec JM, Bennett RL, Carey ME, et al. Projecting the supply and demand for certified genetic counselors: a workforce study. J Genet Couns. 2018;27:16-20.

3. De Castro MJ, Turner CE. Military genomics: a perspective on the successes and challenges of genomic medicine in the armed services. Mol Genet Genomic Med. 2017:5:617-620.

4. Hellwig LD, Turner C, Manolio TA, et al. Return of secondary findings in genomic sequencing: military implications. Mol Genet Genomic Med. 2019;7:e00483.

5. Smith DJ, Bono RC, Slinger BJ. Transforming the Military Health System. JAMA. 2017;318:2427-2428.

6. Vassy JL, Lautenbach DM, McLaughlin HM, et al. The MedSeq Project: a randomized trial of integrating whole genome sequencing into clinical medicine. Trials. 2014;15:85.

7. Vassy JL, McLaughlin HL, MacRae CA, et al. A one-page summary report of genome sequencing for the healthy adult. Public Health Genomics. 2015;18:123-129.

8. Vassy JL, Christensen KD, Schonman EF, et al. The impact of whole genome sequencing on the primary care and outcomes of health adult patients: a pilot randomized trial. Ann Int Med. 2017;167:159-169.

9. Christensen KD, Vassy JL, Jamal $L$, et al. Are physicians prepared for whole genome sequencing? A qualitative analysis. Clin Genet. 2016;89:228-234.

10. Brothers $K B$, East KM, Kelley WV, et al. Eliciting preferences on secondary findings: the Preferences Instrument for Genomic Secondary Results. Genet Med. 2017;19:337-344.

11. Jorde L, Varey J, Bamshad M, White R. Medical genetics. St Louis: Mosby; 2006.
12. Baron RJ. What's keeping us so busy in primary care? A snapshot from one practice. N Engl J Med. 2010;362:1632-1663.

13. Gray SW, Hicks-courant K, Cronin A, Rollins BJ, Weeks JC. Physicians' attitudes about multiplex tumor genomic testing. J Clin Oncol. 2014;32: 1317-1323.

14. Kaphingst KA, Kreuter MW, Casey $C$, et al. Health Literacy INDEX: development, reliability, and validity of a new tool for evaluating the health literacy demands of health information materials. J Health Commun. 2012; 17(Suppl 3):203-221.

15. Ostergren JE, Gornick MC, Carere DA, et al. How well do customers of direct-to-consumer personal genomic testing services comprehend genetic test results? Findings from the Impact of Personal Genomics Study. Public Health Genomics. 2015;18:216-224.

16. Bowling BV, Acra EE, Wang L, et al. Development and evaluation of a genetics literacy assessment instrument for undergraduates. Genetics. 2008;178:15-22.

17. Kaphingst KA, Facio FM, Cheng MR, et al. Effects of informed consent for individual genome sequencing on relevant knowledge. Clin Genet. 2012; 82:408-415.

18. R: A language and environment for statistical computing. Vienna, Austria: R Foundation for Statistical Computing; 2013.

19. McLaughlin HM, Ceyhan-Birsoy O, Christensen KD, et al. A systematic approach to the reporting of medically relevant findings from whole genome sequencing. BMC Med Genet. 2014;15:134-146.

20. US Department of Health and Human Services. Protection of human subjects. 45 CFR $\S 46$.

21. NEJM Catalyst. What is patient-centered care? https://catalyst.nejm.org/ doi/full/10.1056/CAT.17.0559. Accessed 29 Nov 2019.

22. American College of Cardiology. Pediatric familial hypercholesterolemia: Expert analysis. https://www.acc.org/latest-in-cardiology/articles/2015/11/ 24/14/32/pediatric-familial-hypercholesterolemia. Accessed 29 Nov 2019.

23. Tercanli S, Miny P, Siebert MS, Hösli I, Surbek DV, Holzgreve W. Fanconi anemia associated with increased nuchal translucency detected by first-trimester ultrasound. Ultrasound Obstet Gynecol. 2001;17:160-162.

24. Tandy-Connor S, Guiltinan J, Krempely K, et al. False-positive results released by direct-to-consumer genetic tests highlight the importance of clinical confirmation testing for appropriate patient care. Genet Med. 2018;20:1515-1521.

25. Lent RC. Overcoming textbook fatigue: 21st century tools to revitalize teaching and learning. Alexandria, VA: ASCD; 2012.

26. Miller CE, Krautscheid P, Baldwin EE, et al. Genetic counselor review of genetic test orders in a reference laboratory reduces unnecessary testing. Am J Med Genet A. 2014;164A:1094-1101.

27. Kotzer KE, Riley JD, Conta JH, Anderson CM, Schahl KA, Goodenberger $\mathrm{ML}$. Genetic testing utilization and the role of the laboratory genetic counselor. Clin Chim Acta. 2014;427:193-195.

\section{MilSeq Project}

\section{Megan D. Maxwell, MS, LCGC $\oplus^{1,2,3,4,5}$, Rebecca Hsu, BA ${ }^{6}$, Jill O. Robinson, MA ${ }^{6}$, Stacey Pereira, PhD ${ }^{6}$, Cubby L. Gardner, PhD, FNP-C $C^{7}$, Robert C. Green, MD, MPH ${ }^{4,5,8}$ and Mauricio De Castro, MD, FACMG ${ }^{9}$}

\footnotetext{
${ }^{1}$ University Health System, San Antonio, TX, USA; ${ }^{2}$ University of Texas Health Science Center, San Antonio, TX, USA; ${ }^{3}$ Lackland Air Force Base, San Antonio, TX, USA; ${ }^{4}$ Brigham and Women's Hospital, Boston, MA, USA; ${ }^{5}$ Harvard Medical School, Boston, MA, USA; ${ }^{6}$ Baylor College of Medicine, Houston, TX, USA; ${ }^{7}$ US Army Medical Research and Development Command, Fort Detrick, MD, USA; ${ }^{8}$ Broad Institute of MIT and Harvard, Cambridge, MA, USA; ${ }^{9}$ Air Force Medical Genetics Center, Keesler Air Force Base, Biloxi, MS, USA. A full list of members and their affiliations appears in the Supplementary Information.
} 